\title{
A NOTE ON WALLMAN EXTENDIBLE FUNCTIONS
}

\author{
DARRELL W. HAJEK
}

\begin{abstract}
It is known that any continuous function into a $T_{4}$ space has a unique continuous Wallman extension, and that any continuous Wallman extension of a continuous function with a $T_{3}$ range must be unique. We show that for any $T_{3}$ space $Y$ which is not $T_{4}$ there exists a $T_{3}$ space $X$ and a continuous function $f: X \rightarrow Y$ which has no continuous Wallman extension.
\end{abstract}

In this paper we will consider only $T_{1}$ spaces. In [2] it is shown that if $Y$ is a $T_{3}$ space and $f: X \rightarrow Y$ is a continuous function having a continuous Wallman extension $f^{*}: W(X) \rightarrow W(Y)$ then the extension is unique. Furthermore it follows immediately from the fact that if $Y$ is $T_{4}$ then $W(Y)$ is $T_{2}$ and from the Taimanov theorem (see [1, p. 110]) that if $Y$ is $T_{4}$ then any continuous function $f: X \rightarrow Y$ has a continuous Wallman extension, and so it is natural to ask whether the condition that $Y$ be $T_{4}$ can be relaxed. In this paper we show that, if consideration is restricted to $T_{3}$ spaces, the answer is no.

Recall that for a given space $X$ the Wallman compactification $W(X)$ is the collection of all ultrafilters in the lattice of closed subsets of $X$ given the topology generated by the collection of all sets of the form $C(A)=$ $\{u \in W(X): A \in u\}$, where $A$ is closed in $X$ as a base for the closed sets.

The function $\varphi_{X}: X \rightarrow W(X)$ defined by $\varphi_{X}(x)=\{A: A$ closed in $X$ and $x \in A\}$ is a dense embedding of $X$ in $W(X)$. A Wallman extension of a function $f: X \rightarrow Y$ is a function $f^{*}: W(X) \rightarrow W(Y)$ such that $f^{*} \circ \varphi_{X}=$ $\varphi_{Y} \circ f$.

THEOREM. Let $Y$ be a $T_{3}$ space. Then, unless $Y$ is $T_{4}$, there is a $T_{3}$ space $X$ and a continuous function $f: X \rightarrow Y$ which has no continuous Wallman extension.

Proof. In [2] it was proved that if $T$ is $T_{3}$, then, given any continuous function $g: Z \rightarrow T$ which has a continuous Wallman extension $g^{*}: W(Z) \rightarrow$ $W(T)$, for each $u \in W(Z),\left\{g^{*}(u)\right\}=\bigcap\left\{C\left(\operatorname{cl}_{T}(g[A])\right): A \in u\right\}$. Suppose now

Received by the editors April 29, 1973 and, in revised form, July 11, 1973.

AMS (MOS) subject classifications (1970). Primary 54C20; Secondary 54B99, 54D10, 54D15, 54D35.

Key words and phrases. Wallman extension, Wallman extendible function, WOfunction.

(c) American Mathematical Society 1974 
that $Y$ is not $T_{4} . W(Y)$ is not Hausdorff; so there exist two points $u, v \in W(Y)$ which cannot be separated by disjoint open sets. Let $\mathcal{O}$ denote the set $\left\{\varphi_{\bar{Y}}^{-1}[U \cap V]: U, V\right.$ open in $W(Y), u \in U$, and $\left.v \in V\right\}$. We denote by $X$ the product space $X\{O: O \in \mathcal{O}\}$ and by $q$ the projection of $X$ onto $\varphi_{Y}^{-1}[W(Y) \cap W(Y)]=Y$. For each $P \in \mathcal{O}$ we define $A(P)$ to be $\left\{\left(y_{O}\right)_{O \in \mathcal{O}} \in\right.$ $\left.X: P \subseteq O \Rightarrow y_{P}=y_{O}\right\}$. It is immediate that $\{A(P): P \in \mathcal{O}\}$ is a filterbase in the lattice of closed subsets of $X$, and, hence, must be contained in some $w \in W(X)$. Suppose $q$ were to have a continuous Wallman extension $q^{*}$. If $q^{*}(w) \neq u$ there is some $K \in w$ such that $u \notin C\left(\operatorname{cl}_{Y}(q[K])\right)$. However

and

$$
q_{Y}^{-1}\left[W(Y) \sim C\left(\operatorname{cl}_{Y}(q[K])\right)\right]=Y \sim \operatorname{cl}_{Y}(q[K]) \in \mathcal{O}
$$

$$
A\left(Y \sim \operatorname{cl}_{Y}(q[K])\right) \subseteq q^{-1}\left[Y \sim \operatorname{cl}_{Y}(q[K])\right] ;
$$

so, since $A\left(Y \sim \operatorname{cl}_{Y}(q[K])\right)$ must have nonempty intersection with $K$, $q^{*}(w)$ must be $u$, but, since precisely the same argument can be used to show $q^{*}(w)=v$, we must conclude that $q$ has no continuous Wallman extension.

\section{REFERENCES}

1. R. Engelking, Outline of general topology, PWN, Warsaw, 1965; English transl., North-Holland, Amsterdam; Interscience, New York, 1968. MR 36 \#4508; 37 \#5836.

2. D. Hajek, $A$ characterization of $T_{3}$ spaces, Indiana Univ. Math. J. 23 (1973), 23-25.

3. H. Wallman, Lattices and topological spaces, Ann. of Math. 89 (1938), 112-126.

Department of Mathematics, University of Puerto Rico, Mayaguez, Puerto RiCO 00708 